Aim Re-audit, ten year interval using the same survey to assess whether there had been improvement in practise and also reevaluate patients' preferences.

Method Eight question survey in our home ventilation clinic selecting 20 patients with severe COPD on domiciliary non-invasive ventilation. Results were compared to those of 19 similar patients from 2002. Statistical differences were explored with the fishers-exact test. Results The proportion of patients who had been asked about CPR/ETV preferences had not improved between 2002 and 2012; $4 / 19(21.1 \%)$ in 2002 and $7 / 20(35 \%)$ in $2012(p=0.54)$ and remained unacceptably high. The majority of patients wanted more opportunity to discuss their preferences; $17 / 19$ (89.5\%) in 2002 and $11 / 20$ $(55.0 \%)$ in 2012 ( $p=0.038)$. In both 2002 and 2012, patients' preferences for CPR/ETV were influenced by the likelihood of survival but a significant minority wished to receive these therapies even with a quoted chance of survival $<1 \%$ or negligible (figure 1 ).

Discussion Despite ten years awareness of the issue, our institution had failed to significantly improve CPR/ETV discussions in COPD patients attending the home ventilation clinic. We found that people frequently had their own views regarding CPR/ETV and most people would like the opportunity of further discussion. The trust wide use of DNAR forms has not sufficiently improved practise. We believe that the use of a trust wide resuscitation status form might improve this outcome.

1. Divo M et al. Comorbidities and Mortality Risk in Patients with COPD. Am J Respir Crit Care Med. April 2012.



Abstract P231 Figure 1

\section{P232 COMMUNICATION AND END OF LIFE CARE (EOLC) IN PEOPLE WITH RESPIRATORY DISEASE}

doi:10.1136/thoraxjnl-2012-202678.293

${ }^{1} \mathrm{C}$ Hodgekiss, ${ }^{2} \mathrm{~A}$ Edwards, 'IJ Clifton. 'St James's University Hospital, Leeds, UK; ${ }^{2}$ Wheatfields Hospice, Leeds, UK

Background Leeds Teaching Hospitals Trust (LTHT) is a flagship trust for the National End of Life Care (EoLC) strategy. Key areas of improvement in EoLC have been identified, including identification of people approaching end of life and communication of this to the individual, family and primary care colleagues.

The Gold Standards Framework is a national systematic evidence based approach to optimising EoLC. This retrospective study reviewed all deaths during in-patient stay and within 28 days of discharge from respiratory medicine.

Methods All in-patient deaths or deaths within 28 days of discharge from hospital under the care of a respiratory physician at LTHT between April and September 2011 were reviewed. All communication with primary care in the preceding 12 months was reviewed.
Results 144 individuals died on respiratory wards, median (range) age of 76 (18-96) years with the majority having a length of stay over 8 days. 42 individuals died within 28 days of discharge from a respiratory ward, median (range) age of 71 (42-87) years. The commonest cause of death was pneumonia and lung malignancy for inpatient and post-discharge deaths respectively. $23.8 \%$ and $83 \%$ of in-patient and post-discharge deaths respectively had documented communication with primary care about a palliative intent to care, the majority of these had a diagnosis of thoracic malignancy. Within the 12 months pre-death all patients had evidence that EoLC may have been appropriate to consider.

Conclusions Palliative communication with primary care was made for some individuals, mostly with lung malignancy. This probably reflects more predictable disease trajectory and MDT decisions of "best supportive care". Lack of confidence around predicting terminal disease in other respiratory conditions, particularly those such as COPD which are prone to exacerbations, may account for the differences in rates of communication of palliative care approaches in these disease groups.

A key driver for the implementation of high quality EoLC for patients with respiratory disease is recognition of patients approaching the end of life and communication with the individual, family and primary care to ensure that the patient's wishes for EoLC are identified and supported.

\section{P233 DEATH AND THE RESPIRATORY PHYSICIAN: CHALLENGES TO PROVIDING OPTIMAL END-OF-LIFE CARE BY GENERALISTS}

doi:10.1136/thoraxjnl-2012-202678.294

LJ Smith, S Vergnaud, H Wright, C Bates. Queens Hospital, Romford, United Kingdom

Background Surveys show most patients want to die at home. However $53 \%$ of all UK deaths occur in hospital. Patients with chronic respiratory disease are more likely to die in hospital (66\% of COPD deaths) yet hospital end of life care is often poor. Clinicians are advised to use the 'surprise question' to identify patients that need advance care planning (ACP). Do not attempt resuscitation (DNAR) orders (evidence of ACP) are often not completed. Barriers previously identified include: lack of training, time, appropriate opportunity and experience; personal discomfort; and perceived lack of patients'/carers' understanding.

Objective We investigated experiences, beliefs and attitudes of doctors in a district general hospital towards end of life care, focusing on issues relevant to Respiratory patients.

Methods Clinicians of varying grades were invited to complete a multiple-choice questionnaire during 'Dying Matters Awareness Week 2012'

Results Amongst the 73 doctors (49\% male) there was a high degree of confidence (eg $76 \%$ agreed or strongly agreed that they were comfortable talking to patients/relatives about death and dying).However this did not correlate with familiarity with the 'surprise question' (23\% said they were familiar but only $3 \%$ gave a correct response), or knowledge of the most distressing end of life symptom (only $18 \%$ identified shortness of breath correctly), or knowledge of the patient group with the highest unmet palliative care needs (only 23\% identified patients with Respiratory diseases). $40 \%$ believed "palliative care is a specialist skill that should be delivered by specialists".

Discussion There is a pressing need for greater expertise in general palliative care amongst hospital doctors. Patients with COPD and other progressive respiratory conditions have extensive palliative care needs. Accurate prognostication is challenging; the surprise question is useful in prompting ACP. DNAR decisions and ACP should not be left to the last days of life. We identified a mismatch 
between knowledge and confidence levels in our sample. Lack of awareness of their need for education may represent a barrier to doctors accessing training. Respiratory physicians are well placed to lead improvements in ACP and general palliative care in hospital.

\section{Respiratory education and training}

\section{P234 TRAINING DEFICIENCIES AND LACK OF CONFIDENCE AROUND KNOWLEDGE IN PRIMARY CARE NURSES TREATING ASTHMA AND COPD PATIENTS}

doi:10.1136/thoraxjnl-2012-202678.295

${ }^{1} \mathrm{AG}$ Davison, ${ }^{2} \mathrm{~L}$ Jongepier. 'East of England Pharmaceutical Alliance; ${ }^{2} \mathrm{NHS}$ Midlands and East, Cambridge, England

There has been an increasing shift of respiratory care from secondary to primary. We have undertaken a training needs analysis by questionairre of primary care nurses who treat patients with Asthma and COPD in all 511 practises in the East of England. 63\% responded. Nurses were asked to grade their confidence levels from high (5) to low (1) in aspects of Asthma and COPD. Chi-square was used for statistical analysis.

Results 1 ASTHMA. 63\% had a diploma. 90\% followed BTS/SIGN guidelines. There was a high confidence level in $27 \%$ for differential diagnosis, $52 \%$ for inhaler devices and how to use them, $24 \%$ for interpretation of spirometry, 39\% for emergency treatment, $22 \%$ for dealing with children 5-12 years old. High confidence was significantly greater $(<0.001)$ in those with an asthma diploma.

2. COPD. $35 \%$ had a diploma. $82 \%$ followed NICE or GOLD guidelines. $76 \%$ ran clinics. There was a high confidence in $37 \%$ diagnosis, $37 \%$ for management and monitoring, $26 \%$ for interpreting spirometry, $32 \%$ in emergency treatment. High confidence was significantly greater in those with a COPD diploma. Nurses were asked if they were aware of services and confident how to refer; $94 \%$ were for smoking cessation, $55 \%$ for spirometry, $35 \%$ for oxygen assessment, $63 \%$ for pulmonary rehabilitation. The level of awareness was significantly higher for the latter two in those with a diploma. 3. ONGOING TRAINING OPPORTUNITIES Nurses were asked where and how often training was accessed on a scale $1-5$. The most frequent (5) was self-directed learning in 37\% and from the pharmaceutical industry $17 \%$; the least (1) were time to learn $46 \%$, monitoring with a practice expert $45 \%$, secondary care provider $43 \%$.

Conclusions This study has revealed serious deficiencies in training with many having no diploma. The benefit of having a diploma is shown in greater confidence in knowledge and in awareness of services and how to refer. On-going professional education is haphazard. If increasing care is going to be successfully transferred into primary both these issues must be addressed.

\section{P235 THE BRISTOL COPD KNOWLEDGE QUESTIONNAIRE (BCKO): ASSESSING THE KNOWLEDGE OF HEALTHCARE PROFESSIONALS INVOLVED IN THE DELIVERY OF COPD SERVICES}

doi:10.1136/thoraxjnl-2012-202678.296

K Edwards, S Singh. University Hospitals of Leicester NHS Trust, Leicester, United Kingdom

Introduction Healthcare professionals (HCPs) involved in the delivery of COPD services and self-management interventions require appropriate knowledge to inform patients about their condition. Although patient knowledge is often assessed to encourage patient education and self-management, little is done to assess the knowledge of HCPs providing disease information to patients and to establish whether HCPs have a broad spectrum of knowledge to effectively support disease management.
Objective To assess the knowledge of HCPs involved in the delivery of COPD services.

Method The Bristol COPD Knowledge Questionnaire (BCKQ) was distributed to 108 HCPs working in primary and secondary care COPD services (Coventry, Leicester, Lothian, Nottingham and Northampton) to assess knowledge. The $\mathrm{BCKO}$ is primarily an outcome measure for patient knowledge (White et al. 2006). It is a multiple choice questionnaire containing 13 topics, each with five statements giving a total of 65 questions for which there is a right or wrong answer. Positive scoring was used with a mark being given for a correct answer. Incorrect responses indicate a knowledge deficit.

Results The overall findings revealed that HCPs had a mean score of $50(77 \%)$ (minimum 24, maximum 62). The results from the $\mathrm{BCKQ}$ revealed particular gaps in knowledge in the breathlessness topic with a mean score of 3.3 (66\%) (minimum 1, maximum 5) and across the medication topics, particularly inhaled steroids with only a mean score of 2.7 (54\%) (minimum 0, maximum 5) of participants providing correct answers.

Conclusion The HCPs involved in the delivery of these COPD services had particular gaps in knowledge around breathlessness and medications. HCP gaps in knowledge could inadvertently impact patient knowledge and understanding of their condition and subsequently the ability of patients to effectively self-manage their COPD. Identifying gaps in knowledge can encourage HCP education and training to enhance HCP knowledge and subsequent patient care.

1. White, R. Walker, P. Roberts, S. Kalisky, S and White, P (2006) 'Bristol COPD Knowledge Questionnaire (BCKO): testing what we teach patients about COPD'. Chronic Respiratory Disease 3: 123-131.

\section{P236 THE USE OF LOCAL ANAESTHESIA FOR ARTERIAL BLOOD GAS SAMPLING - A MULTICENTRE SURVEY}

doi:10.1136/thoraxjnl-2012-202678.297

${ }^{1}$ F Khan, ${ }^{2} \mathrm{~A}$ D'Silva, ${ }^{3} \mathrm{~S}$ Ahmed, ${ }^{4} \mathrm{E}$ Patel, ${ }^{5} \mathrm{~S}$ Hassan, ${ }^{1} \mathrm{ARC}$ Patel. ${ }^{1}$ Whipps Cross University Hospital, London, UK; '2Southend University Hospital, Southend on Sea, UK; ${ }^{3}$ Royal London Hospital, London, UK; ${ }^{4}$ Homerton University Hospital, London, UK; ${ }^{5}$ Newham University Hospital, London, UK

Background BTS guidance for Emergency Oxygen Use recommends that local anaesthesia should be used for all routine arterial blood gas (ABG) sampling [1]. Intradermal and/or subcutaneous local anaesthetic (LA) via small gauge needle has been shown to reduce pain associated with the procedure by more than half [2]. We aimed to quantify the prevalence of this practise and ascertain potential barriers.

Methods 160 FY1 doctors with at least eight months working experience, from five hospitals in London were given a ten-item anonymised questionnaire to measure practise and opinions regarding local anaesthesia before ABG sampling.

Results All 115 respondents (72\% response rate) performed $A B G$ sampling regularly, with $84 \%$ doing so at least weekly.

Only $27 \%$ of respondents had ever used intradermal and/or subcutaneous LA before ABG sampling, although only $5 \%$ did this regularly.

The commonest needles used were 25 gauge (orange) (49\%), 28 gauge (insulin needle) (18\%), and 23 gauge (blue) (16\%).

$70 \%$ of respondents had never used LA of any kind for ABG sampling. Topical LA use was rare.

$14 \%$ of respondents had never heard of LA used for ABG sampling; $24 \%$ were unfamiliar with how to do it; $14 \%$ believed it was potentially dangerous; $34 \%$ claimed not to have time, $21 \%$ believed that LA would not reduce the overall pain of the procedure (Figure 1).

Allen's test was usually perfomed by only $25 \%$ of doctors before ABG sampling. 